\title{
Análisis de aprendizaje conceptual empleando CDFs: documentos con un formato computable
}

| Conceptual learning analysis using CDFs: documents in a computable format |

\author{
Enrique Vílchez Quesada \\ enrique.vilchez.quesada@una.ac.cr \\ Universidad Nacional de Costa Rica \\ Heredia, Costa Rica
}

\author{
Juan Félix Ávila Herrera \\ delagarita@gmail.com \\ Universidad Nacional de Costa Rica \\ Heredia, Costa Rica
}

Resumen: Este artículo comparte los principales hallazgos de una investigación realizada en el contexto del curso MAT030 Matemática para Informática de la carrera Ingeniería en Sistemas de Información de la Universidad Nacional de Costa Rica, que tuvo como objetivo general analizar el impacto de la integración pedagógica del uso de un tipo especial de documento denominado CDF por sus siglas en inglés: computable document format (documento con un formato computable). La investigación se sustentó en utilizar ciento treinta aplicaciones CDFs diseñadas por los autores, junto a un libro de texto, como recursos didácticos para apoyar la docencia y el autoaprendizaje de los contenidos de esta asignatura. Se empleó para ello una metodología cuantitativa a través de dos grupos experimentales con 27 participantes y dos grupos de control durante el I semestre del año 2020, ejecutándose cinco pruebas de hipótesis para analizar la existencia o no, de diferencias en la comprensión conceptual de los ejes temáticos: lógica proposicional, teoría de números, teoría de conjuntos, inducción y progresiones, y teoría de funciones. Además, se aplicó un cuestionario a los grupos experimentales para rescatar sus percepciones más importantes sobre la experiencia didáctica y la calidad de los materiales diseñados. Los resultados obtenidos en las pruebas de hipótesis evidencian una mejoría en la comprensión conceptual y, por ende, en los procesos de aprendizaje dentro de los grupos experimentales donde las aplicaciones CDFs jugaron un rol preponderante en el desarrollo de formas de razonamiento, procesos de análisis, autoevaluación, y visualización de conceptos, propiedades y algoritmos. También, los grupos experimentales mostraron un rendimiento académico más favorable. La investigación constata cómo el uso adecuado de la tecnología educativa constituye un instrumento que potencializa la adquisición de destrezas de aprendizaje y habilidades lógicas en la educación matemática, y por lo tanto, cómo esta puede ofrecer una alternativa viable para promover una combinación efectiva con un enfoque no tradicional.

Palabras Clave: CDFs, matemática para informática, enseñanza, aprendizaje, análisis.

Abstract: This article shares the main findings of research carried out in the context of MAT030 Math for Informatics, a course of the Information Systems Engineering, an undergraduate program at the National University of Costa Rica. The main goal of this research was to analyze the impact of the pedagogical integration of the use of a special type of document called CDF (for its acronym in English: computable document format). The research was based on using one-hundred and thirty CDF applications designed by the authors, along with a textbook, as didactic resources to support teaching and self-learning of the contents of MAT030 course. For this, a quantitative methodology was used 
through two experimental groups with 27 participants and a control group during the first semester of 2020. Five hypothesis tests were executed to analyze the existence of differences in the conceptual understanding of the thematic axes: propositional logic, number theory, set theory, math induction and progressions, and function theory. In addition, a questionnaire was applied to the experimental groups to obtain their most important perceptions about the experience and the quality of the materials designed. The results obtained in the hypothesis tests show an improvement in conceptual understanding and, therefore, in the learning processes within the experimental groups, where the CDF applications played a preponderant role in the development of ways of reasoning, analysis processes, self-evaluation, and visualization of concepts, properties, and algorithms. Also, the experimental groups showed a more favorable academic performance. The research confirms how the appropriate use of educational technology constitutes an instrument that enhances the acquisition of learning skills and logical skills in mathematics education and, therefore, how it can offer a viable alternative to promote an effective combination with a non-traditional approach.

Keywords: CDFs, computer mathematics, teaching, learning, analysis.

\section{Introducción}

El curso MAT030 Matemática para Informática, impartido en la carrera Ingeniería en Sistemas de Información, adscrita a la Escuela de Informática de la Universidad Nacional de Costa Rica (UNA) presenta, ya desde hace varios años, una situación preocupante debido a la baja promoción, la deserción y a la repitencia. Según las estadísticas de rendimiento académico de la cátedra correspondientes al I Ciclo lectivo 2017, solo un 42.03 \% de alumnos matriculados lograron aprobar el curso.

En esta materia se ha venido utilizando una metodología de enseñanza y aprendizaje tradicional, esto es, empleando varios textos de clase, listas de ejercicios y apuntes informales además de la tradicional clase magistral. En virtud de que este enfoque no estaba ofreciendo alternativas metodológicas que propiciaran un acompañamiento cognitivo apropiado, resultaba claro, que era necesario buscar un nuevo abordaje didáctico que ofreciera oportunidades reales a una población poco homogénea, esto debido a sus características socio-geográficas amén de la multiplicidad de deficiencias en conocimientos previos (Vílchez y Ávila, 2021). Es por todas estas razones que se decidió recurrir al desarrollo de CDFs (documentos con un formato computable) como un instrumento que pudiera coadyuvar positivamente en un cambio metodológico sustancial.

Los CDFs son aplicaciones en software que se desarrollan mediante la herramienta Wolfram Mathematica. Tales aplicaciones permiten a los usuarios interactuar (con ellas) sin tener que dominar el lenguaje de programación Mathematica, normalmente requerido para aprovechar esta plataforma y cuya curva de aprendizaje es generalmente lenta.

Fue con base en este contexto que se decidió proponer un proyecto de investigación, auspiciado por la UNA, y con el fin de desarrollar para MAT030 un conjunto de CDFs, un libro de texto (Vílchez y Ávila, 2020) y una colección de videos, entre otros productos. La intención de los autores consistió en brindar una nueva orientación de acción didáctica y que estuviera en consonancia con una matemática educativa donde se valore la experimentación, la observación, el análisis y la interacción dinámica como un proceso metodológico en el que el rol del estudiante es más activo y gestor fundamental de su propio avance.

En este trabajo se detalla la experiencia de apoyar, con los recursos tecnológicos descritos con anterioridad, un curso de matemática introductoria para computación. Es justo indicar que, al inicio del proyecto, no existía en la academia dentro de la Universidad Nacional de Costa Rica, una propuesta con herramientas similares a las aquí planteadas en asignaturas de matemática para informática. 


\section{Marco teórico}

En muchos centros de estudio, la educación matemática se ha tratado de concebir como una colección de algoritmos (o recetas) que se deben memorizar y aplicar. Algunos ejemplos de estos son el uso de la fórmula general para resolver ecuaciones cuadráticas, las listas de derivadas e integrales, las leyes lógicas, el método de Gauss-Jordan. Resulta que muchas veces el aprendizaje de estos procedimientos se vuelve un fin en sí mismo y se puede caer en algo semejante a la famosa paradoja del cuarto chino usada en el área de la Inteligencia Artificial propuesta por John Searle (Penrose, 1991). La paradoja consiste en que, aunque los estudiantes exhiban un comportamiento que pareciera indicar que dominan la materia, en el fondo simplemente están respondiendo (de forma predeterminada) a ciertos estímulos. La consecuencia de esto es que poco después de acabado un curso de matemática, el estudiante olvida casi todo lo "aprendido".

En estos enfoques tradicionales el profesor tiene el rol principal, es de alguna forma el "dueño" del conocimiento y las cosas deben hacerse a su estilo. Este enfoque puede inhibir al estudiante y muchas veces limitar su capacidad de experimentación y frenar el descubrimiento de algunos resultados matemáticos por su propia cuenta (Artigue, 2013).

Por otro lado, algunas veces se ha caído en el error de identificar o confundir el dominio de los conocimientos matemáticos con la aprobación de un cierto curso o examen. El problema se agrava cuando en las materias subsiguientes no se hace ningún tipo de prueba de diagnóstico y el estudiante continúa "avanzando", tratando de remediar, por su cuenta, una serie de deficiencias. Esta situación se vuelve algunas veces una "bola de nieve" que sirve de lastre para el alumno y es la razón de muchas repitencias y deserciones (Gerver, 2013).

Una educación matemática de calidad, por consiguiente, no puede desestimar el desarrollo de las competencias más relevantes basadas en la experimentación, el descubrimiento de resultados, la verificación, la prueba rigurosa y el afán de generalizar lo aprendido tanto como se pueda. Es bien sabido que una buena forma de aprender un concepto (casi en cualquier área) es mantener viva la pregunta ¿qué pasa si hago un cambio en esto? Sin embargo, algunos docentes, por su propia comodidad, tratan de estructurar los temas de manera que el estudiante se limite a un cierto rango de situaciones. Si este es el caso, se induce en el alumno a adoptar por una actitud semipasiva que a lo mejor se vuelve atractiva solamente porque el conjunto de problemas asignados es susceptible de venir en la próxima evaluación (Cantoral, 2016).

Es bajo esta convicción de querer poner en las manos de los estudiantes herramientas que les permitan romper con este paradigma de enseñanza tradicional, que los autores del presente artículo asumieron este importante reto. Para ello, se integró el uso pedagógico de CDFs orientados al curso MAT030 mediante un libro de texto con posibilidades interactivas, proporcionando de esta manera valiosas oportunidades de transformación en el accionar didáctico.

En los grupos experimentales participantes de esta investigación, se asumió una postura epistemológica vinculada con el constructivismo y el uso de las tecnologías de información y comunicación como puentes entre el conocimiento matemático y la reflexión continua hacia el replanteamiento de significados, contenidos y aprendizajes en el marco de una práctica educativa en evolución y mejoramiento (Castillo, 2008).

Es justo mencionar que, además de la complejidad en la enseñanza y el aprendizaje de la matemática, los actores de este proceso deben lidiar con una premisa que es vox populi, a saber, "la matemática es difícil" por sí misma. Por supuesto que existen conceptos y procedimientos matemáticos difíciles, pero los temas abordados en la educación preuniversitaria son en buena parte de "fácil digestión". Cuando los conceptos matemáticos se explican planteando su relación con problemas reales (utilizando elementos del entorno del estudiante) y mostrando, por ejemplo, las ventajas de utilizar variables o parámetros en lugar de casos particulares, el proceso tiende a ser de mejor aceptación por parte del alumno (Resnick y Ford, 1990). Sin embargo, cuando el docente se empecina en mantener un nivel formal, libre de interpretaciones intuitivas, los estudiantes tienden a sentirse frustrados pues recae en 
ellos la tarea de buscar situaciones cotidianas que expliquen o den sentido, de alguna forma, al tema que se estudia.

La clave reside, entonces, en proveer espacios de aprendizaje inclusivos que brinden igualdad de oportunidades, experimentación, comprobación y, por qué no, vínculos socioafectivos, producto de una valoración hacia la experimentación y la generación de ideas que fomenten la creatividad personal y colectiva (Vílchez, 2014). Es debido a esto que se consideró, en esta investigación, que el uso educativo apropiado de los CDFs en MAT030 surtiría decisivamente un efecto de mejoría en la enseñanza de sus contenidos. El empleo de la tecnología educativa puede contribuir favorablemente en la consecución de enfoques de enseñanza más participativos y de naturaleza constructiva por descubrimiento (García, 2014). El presente trabajo partió de esta premisa y durante su desarrollo se fueron recabando datos que permitieron medir, de manera científica, el impacto de empleo de la tecnología de CDFs en el proceso de transferencia, construcción y análisis del conocimiento dentro de los entornos educativos asociados a MAT030.

A este respecto, el fundamento pedagógico que caracterizó en la presente propuesta la finalidad didáctica de los $\mathrm{CDFs}$, cobra una postura también constructivista donde estas herramientas de software otorgan a los alumnos formas de representación diversa de los contenidos, articulando un engranaje de objetos visuales y de interacción dinámica hacia la elaboración y comprensión de definiciones y métodos de resolución de problemas (Ávila y Vílchez, 2019).

El uso de CDFs si bien es cierto que abre un interesante abanico de posibilidades pedagógicas, tampoco es una opción que resuelve todas las dificultades (Vílchez y Ávila, 2021). Se pueden construir CDFs que generen grandes cantidades de ejercicios (con respuesta incluida) y que permitan aprovechar la visualización de objetos matemáticos en casi cualquier tipo de computador o dispositivo móvil (Vílchez y Ávila, 2019). Es posible también crear CDFs que muestren paso a paso el seguimiento de un procedimiento en particular. Se pueden adjuntar videos educativos que apoyen al estudiante en el estudio independiente, sin embargo, la presencia del docente es irremplazable y debe seguir formando parte importante de cualquier nuevo enfoque que se desee implementar. Por ahora, resulta muy difícil prever todas las posibles complejidades suscitadas en un aula de clase. Esta tarea seguirá dependiendo del profesor y el uso de nuevas herramientas tales como CDFs, videos educativos, entre otras, solo permitirá complementar la atención pertinente de los distintos casos singulares que cotidianamente, caracterizan cualquier grupo de estudiantes y sus necesidades educativas (Robinson, 2015).

\section{Metodología}

La presente investigación tuvo como objetivo analizar la percepción estudiantil en la integración pedagógica del uso de documentos con un formato computable (CDFs) en el contexto de la cátedra MAT030 Matemática para Informática a través de un estudio de caso descriptivo de naturaleza cuantitativa, donde participaron dos grupos experimentales (GE) y dos grupos de control (GC).

Los investigadores de esta propuesta programaron en lenguaje Wolfram durante el año 2019, ciento treinta aplicaciones CDFs disponibles en: https://www.escinf.una.ac.cr/CDF/index.php/matematicapara-informatica. Estos documentos con un formato computable se caracterizaron por estar inmersos en los contenidos del curso MAT030, a saber: lógica formal, teoría de números, teoría de conjuntos, inducción y progresiones, y teoría de funciones. Asimismo, en el año 2019 los investigadores diseñaron un libro de texto teórico-práctico donde incorporaron los CDFs creados. Este texto sirvió como guion didáctico para el tratamiento de un proceso de implementación de usuario. 


\subsection{Grupos participantes y datos recopilados}

En el primer ciclo lectivo del año 2020 los investigadores ejecutaron un proceso de implementación bajo una metodología asistida por computadora para el curso MAT030, empleando como principal referente didáctico un libro de su autoría, titulado: "Matemática para ingeniería mediante el uso de CDFs" (Vílchez y Ávila, 2020). El texto se caracterizó por incorporar en su contenido de manera estratégica ciento treinta documentos con un formato computable relacionados con los temas que aborda la asignatura MAT030.

Los grupos experimentales utilizaron el libro anteriormente señalado y las aplicaciones CDFs durante el primer semestre del año 2020. Al finalizar ese período, se les aplicó a 27 alumnos participantes un cuestionario para determinar su nivel de satisfacción y perspectivas relacionadas con las actividades de enseñanza y aprendizaje llevadas a cabo y la calidad de los CDFs diseñados para la materia (o curso) MAT030.

Los GE estuvieron a cargo de los docentes investigadores y la muestra implicada en la realización del cuestionario, se caracterizó según lo descrito en la Tabla 1.

Tabla 1: Características de la muestra en los grupos experimentales. Fuente: Elaboración propia

\begin{tabular}{ll}
\hline & \multicolumn{1}{c}{ Descripción } \\
\hline Sexo & 21 personas de sexo masculino y 6 de sexo femenino. \\
Edad & 20 tenían un rango de edad entre los 17 y los 19 años, 5 entre \\
& los 20 y 23 años, y 2 con una edad superior o igual a los 24 \\
& y menor a los 27 años. \\
Carga académica & $74.1 \%$ eran alumnos a tiempo completo con respecto a la \\
& cantidad de créditos matriculados y 25.9\% a tiempo parcial. \\
Nivel de repitencia & $70.4 \%$ llevaban la materia MAT030 por primera vez y 29.6\% \\
& la repetían, siendo ésta, la segunda o tercera ocasión. \\
Experiencia en cursos asis- & Un 85.2\%, nunca habían cursado una asignatura apoyada \\
tidos por computadora & con recursos computacionales. \\
\hline
\end{tabular}

Por otra parte, adicionalmente, participaron de este proceso dos grupos de control que fueron asumidos por un académico de la Escuela de Matemática de la Universidad Nacional de Costa Rica, no perteneciente al equipo de investigadores. El método de enseñanza utilizado en estos grupos se fundamentó en una modalidad tradicional con clases magistrales y listas de ejercicios propuestas por la coordinación de la cátedra del curso MAT030, sin emplear ningún recurso adicional, ni software de apoyo.

Las muestras en los GE y los GC fueron probabilísticas y generadas por el azar producido en la matrícula de cada unos de los grupos involucrados.

La metodología de esta pesquisa se fundamentó, además, en la realización de cinco pruebas de hipótesis Mann-Whitney con el objetivo de comparar la comprensión conceptual de diez definiciones entre los grupos experimentales y los grupos de control, y así, determinar si existía una mejor comprensión de esos conceptos en los grupos que sí basaron su aprendizaje, en los documentos con un formato computable.

Finalmente, se recurrió también a los grupos de control, para el suministro de una prueba de hipótesis $t$ de Student orientada a identificar diferencias en cuanto al rendimiento académico mostrado en comparación con los grupos experimentales. 


\subsection{Pruebas de hipótesis: análisis de aprendizaje conceptual}

Los autores de esta propuesta diseñaron cinco instrumentos de comprensión conceptual para el abordaje de cinco pruebas de hipótesis no paramétricas Mann-Whitney en muestras independientes. Por la naturaleza del estudio, resultó apropiada la selección de este tipo de inferencia estadística, según Berlanga y Rubio (2012), y Hurtado y Hurtado (2015), al querer comparar el número de aciertos conceptuales entre los GE y los GC, siendo ambas muestras independientes que no siguen una distribución normal. Además, se deseaba establecer una relación entre la variable cualitativa "método de enseñanza: con o sin CDFs" y la variable cuantitativa ordinal "cantidad de aciertos conceptuales".

Todas las pruebas de hipótesis Mann-Whitney cuyos resultados se comparten en esta sección, tomaron como referencia la siguiente hipótesis nula (Ho) y la siguiente hipótesis alternativa (Ha):

- Ho: el número de conceptos comprendidos no es mayor en alumnos que emplearon documentos con un formato computable en comparación con otros que no los utilizaron.

- Ha: el número de conceptos comprendidos es mayor en alumnos que emplearon documentos con un formato computable en comparación con otros que no los utilizaron.

Los instrumentos de comprensión conceptual incluyeron diez definiciones cada uno, seleccionadas por la experticia de los investigadores en los cinco ejes temáticos principales del curso MAT030.

Se solicitó a los estudiantes participantes en los GE y los GC, describir con sus propias palabras los conceptos que se les presentaron sin necesidad de responder de memoria y contestando de forma individual de acuerdo con lo que sabían en el momento. Se les explicó a los alumnos que esto no afectaría su rendimiento académico en la materia. Los instrumentos se suministraron a los GE y los GC una semana después de haber concluido cada tema.

A razón de la emergencia sanitaria vivida a nivel mundial por el COVID-19, tres de estos instrumentos tuvieron que ser aplicados de manera remota, en presencia de los profesores a su cargo a través de una videollamada, durante el horario de clase en el primer ciclo lectivo 2020. La Tabla 2 señala el momento de suministro de cada uno de los instrumentos conceptuales.

Tabla 2: Semanas de aplicación de los instrumentos conceptuales: primer ciclo 2020. Fuente: Elaboración propia

\begin{tabular}{lc}
\hline & Semana de aplicación \\
\hline Instrumento de lógica proposicional & 4 \\
Instrumento de teoría de números & 6 \\
Instrumento de teoría de conjuntos & 12 \\
Instrumentos de inducción y progresiones & 14 \\
Instrumento del tema de funciones & 19 \\
\hline
\end{tabular}

La información fue tabulada en el software SPSS contando la cantidad de aciertos por estudiante y agrupando cada registro en dos categorías: " 1 = Grupo de control" y "2 = Grupo experimental". Las pruebas de hipótesis Mann-Whitney para muestras independientes, se efectuaron en ese mismo programa de cálculo estadístico, lo cual facilitó la interpretación de los resultados al no tener que recurrir a una tabla de valores críticos de U (estadístico Mann-Whitney), para una prueba unilateral o bilateral bajo un nivel de significancia específico.

Las Tablas 4, 5, 6, 7 y 8 mostradas a continuación, resumen los resultados arrojados por el software SPSS asumiendo un nivel de confianza de un $95 \%$. En ellas, interesa observar la significancia asintótica " $\rho$ " obtenida. El resumen de estos resultados se comparte en la Tabla 3.

De lo anterior, como en todos los casos $\rho$ es menor que 0.05 , se rechaza la hipótesis nula Ho y se acepta la hipótesis de investigación Ha. Es decir, con un error de un $5 \%$, se acepta que: "el número 
Tabla 3: Resumen de los resultados en la significancia asintótica " $\rho$ " obtenida por tema. Fuente: Elaboración propia

\begin{tabular}{lc}
\hline & Significancia asintótica " $\rho$ " \\
\hline Lógica formal & 0 \\
Teoría de números & 0 \\
Teoría de conjuntos & 0.000305 \\
Inducción y progresiones & 0.000003 \\
Teoría de funciones & 0.00001 \\
\hline
\end{tabular}

de conceptos comprendidos es mayor en aquellos estudiantes donde los procesos de enseñanza y aprendizaje estuvieron basados en el uso de documentos con un formato computable".

Se aclara al lector que el número de registros de los grupos experimentales y los grupos de control en cada una de las pruebas de hipótesis por tema, varió en cantidad de acuerdo con el número de estudiantes disponibles al momento de la aplicación del instrumento de comprensión conceptual. Después de cada una de las tablas que siguen, se especifica la cantidad de participantes por categoría.

Tabla 4: Resultados de la Prueba Mann-Whitney: Tema de lógica formal. Fuente: Elaboración propia

\begin{tabular}{lc}
\hline & Significancia asintótica " $\rho$ " \\
\hline Mann-Whitney U & 284.000 \\
Wilcoxon W & 2429.000 \\
$Z$ & -7.122103 \\
Significancia asintótica & $\rho=0.000$ \\
\hline
\end{tabular}

En el tema de lógica formal se utilizaron 65 registros designados como "grupo de control" y 44 registros en la categoría de "grupo experimental".

Tabla 5: Resultados de la Prueba Mann-Whitney: Tema de teoría de números. Fuente: Elaboración propia

\begin{tabular}{lc}
\hline & Significancia asintótica " $\rho$ " \\
\hline Mann-Whitney U & 22.000 \\
Wilcoxon W & 1852.000 \\
$Z$ & -8.683610 \\
Significancia asintótica & $\rho=0.000$ \\
\hline
\end{tabular}

En el tema de teoría de números se emplearon 60 registros en la categoría de "grupo de control" y 45 en "grupo experimental".

Tabla 6: Resultados de la Prueba Mann-Whitney: Tema de teoría de números. Fuente: Elaboración propia

\begin{tabular}{lc}
\hline & Significancia asintótica " $\rho$ " \\
\hline Mann-Whitney U & 207.500 \\
Wilcoxon W & 672.500 \\
$Z$ & -3.610695 \\
Significancia asintótica & $\rho=0.000305$ \\
\hline
\end{tabular}

En el tema de teoría de conjuntos se utilizaron 30 registros de "grupo de control" y 30 de "grupo experimental". 
Tabla 7: Resultados de la Prueba Mann-Whitney: Tema de inducción y progresiones. Fuente: Elaboración propia

\begin{tabular}{lc}
\hline & Significancia asintótica " $\rho$ " \\
\hline Mann-Whitney U & 25.000 \\
Wilcoxon W & 116.000 \\
$Z$ & -4.335948 \\
Significancia asintótica & $\rho=0.000003$ \\
\hline
\end{tabular}

Para el tema de inducción y progresiones se emplearon 13 registros en categoría de "grupo de control" y 25 de "grupo experimental".

Tabla 8: Resultados de la Prueba Mann-Whitney: Tema de teoría de funciones. Fuente: Elaboración propia

\begin{tabular}{lc}
\hline & Significancia asintótica " $\rho$ " \\
\hline Mann-Whitney U & 33.500 \\
Wilcoxon W & 153.500 \\
$\mathrm{Z}$ & -4.120975 \\
Significancia asintótica & $\rho=0.00001$ \\
\hline
\end{tabular}

Finalmente, en el tema de teoría de funciones los registros se distribuyeron en 15 de "grupo de control" y 22 de "grupo experimental".

Se observa cómo a partir del tema de teoría de conjuntos, donde se inició la aplicación de los instrumentos de manera remota, por las circunstancias implicadas a raíz de la pandemia provocada por el COVID-19 y la deserción estudiantil típica en esta clase de cursos, el número de alumnos participantes comenzó a disminuir, pese a ello, esto no quita validez estadística a los resultados y sus interpretaciones ya expuestas.

\subsection{Fiabilidad y validez del cuestionario de percepción estudiantil}

El cuestionario suministrado a una muestra de 27 estudiantes al finalizar el primer ciclo lectivo 2020, se caracterizó por utilizar como modelo en su elaboración, un instrumento validado por expertos en otra pesquisa de la cual formo parte uno de los autores del presente artículo (Vílchez, 2019). Lo anterior justifica la validez de contenido y de constructo del cuestionario empleado.

En cuanto a la fiabilidad del instrumento, este fue sometido a un proceso de confiabilidad alfa de Cronbach en las dos dimensiones respecto a las cuales se subdividió el cuestionario, puntualmente estas son: "actividades asistidas por computadora basadas en el uso de CDFs" y "calidad de los CDFs para la enseñanza y el aprendizaje de la materia MAT030". En ellas, se recurrió al uso de una escala Likert con cinco puntuaciones en el rango: " 1 = Muy de acuerdo", " $2=$ De acuerdo", " $3=$ Mediamente de acuerdo", " 4 = En desacuerdo" y "5 = Muy en desacuerdo". La Tabla 9 muestra los resultados del coeficiente alfa de Cronbach en cada una de estas dimensiones, hallados, mediante el uso del software estadístico SPSS.

Hernández, Fernández y Baptista (2006) señalan que un valor alfa de Cronbach superior a 0.75 evidencia una fiabilidad aceptable, por lo que, para las dimensiones del instrumento en cuestión, los coeficientes alfa de Cronbach de 0.838 y 0.872 (ver Tabla 9) reportan una confiabilidad positiva. 
Tabla 9: Coeficientes alfa de Cronbach. Fuente: Elaboración propia

\begin{tabular}{lcc}
\hline Dimensión & $\begin{array}{c}\text { Valor del coeficiente alfa } \\
\text { de Cronbach }\end{array}$ & $\begin{array}{c}\text { Número de } \\
\text { items }\end{array}$ \\
\hline $\begin{array}{l}\text { Actividades asistidas por computadora basa- } \\
\text { das en el uso de CDFs }\end{array}$ & 0.838 & 12 \\
$\begin{array}{l}\text { Calidad de los CDFs para la enseñanza y el } \\
\text { aprendizaje de la materia MAT030 }\end{array}$ & 0.872 & 18 \\
\hline
\end{tabular}

\subsection{Percepción estudiantil sobre las actividades asistidas por computadora basa- das en el uso de CDFs: ACDFs}

La primera de las dimensiones incluidas en el cuestionario del cual participaron 27 alumnos de los grupos experimentales al finalizar el primer ciclo lectivo 2020, consistió en rescatar la percepción sobre las actividades de enseñanza y aprendizaje implementadas a través del uso de documentos con un formato computable. Estas actividades tomaron como norte pedagógico el libro de texto: "Matemática para ingeniería mediante el uso de CDFs" (Vílchez y Ávila, 2020), además de los documentos con un formato computable diseñados (ver: https://www.escinf.una.ac.cr/CDF/index.php/matematicapara-informatica) y fueron orquestadas por los investigadores de esta propuesta como docentes a cargo de los GE. En la Tabla 10 se comparten los resultados porcentuales obtenidos de la escala Likert utilizada.

Con base en la Tabla 10, se extrajeron como percepciones positivas, aquellas cuya suma porcentual en las puntuaciones " $1=$ Muy de acuerdo" y " $2=$ De acuerdo", dio como resultado un valor mayor o igual a $70 \%$. En función de ello, se destacan como apreciaciones positivas de las ACDFs las siguientes: el trabajo en clase y extraclase complementado con el uso de CDFs fue útil (96.3\%), las ACDFs tuvieron instrucciones claras $(85.2 \%)$, las ACDFs fueron agradables $(85.2 \%)$, la organización del tiempo al realizar las ACDFs fue la adecuada $(77.8 \%)$, las ACDFs fueron formativas en términos de aprendizaje $(85.2 \%)$, las ACDFs llenaron las expectativas del estudiantado $(85.2 \%)$, se tiene en general una percepción positiva de las ACDFs (92.6\%), agradó el uso de software para el curso MAT030 (96.3\%) y se prefiere una metodología no tradicional para la curso MAT030 (74.1\%). Por otra parte, se deducen como percepciones negativas: las ACDFs fueron difíciles $(70.4 \%)$, las ACDFs no fueron divertidas $(59.3 \%)$ y las ACDFs no motivaron el estudio hacia la materia $(40.7 \%)$.

En el cuestionario se incluyeron dos preguntas abiertas consultando a la muestra de estudiantes sus opiniones con respecto a tres fortalezas y tres debilidades de las ACDFs. En cuanto a las debilidades manifestadas por una mayoría de un $70 \%$, se menciona la problemática de su nivel de dificultad aunado a las limitaciones sintácticas sentidas en algunos CDFs con el objetivo de ingresar los datos de procesamiento de la información, al ser necesario conocer ciertos aspectos de usuario vinculados con el software Wolfram Mathematica. También, se señaló la necesidad de contar con un archivo independiente a manera de resumen, donde se describan las 130 aplicaciones CDFs, pues al estar distribuidas en el libro de clase en todos sus capítulos, resultó complejo ubicar documentos específicos con un formato computable. Los investigadores de este trabajo conjeturan que estos dos aspectos tuvieron incidencia sobre las percepciones negativas del estudiantado en los ítems de actividad lúdica y motivación hacia el curso.

Con el objetivo de obtener una medición global de las percepciones de opinión plasmadas en la escala Likert, en la dimensión de "actividades asistidas por computadora basadas en el uso de CDFs", se utilizó el software SPSS para crear una variable denominada MEDIA donde se acumuló la media aritmética " $\mathrm{n}$ " de los registros por individuo, que fueron codificados en valores correspondientes a: " 1 = Muy de acuerdo", " 2 = De acuerdo", " $3=$ Mediamente de acuerdo", " $4=$ En desacuerdo" y " 5 = Muy en desacuerdo". Luego, para analizar el resultado de las medias aritméticas se consideró la siguiente escala de interpretación: $1 \leq n<2$ fue valorada como "Muy favorable", $2 \leq n<3$ se asoció como "Favorable", $3 \leq \mathrm{n}<4$ se clasificó como "Desfavorable" y $4 \leq \mathrm{n} \leq 5$ se valoró en la categoría 
Tabla 10: Porcentajes escala Likert: Actividades asistidas por computadora basadas en el uso de CDFs. Fuente: Elaboración propia

Actividades asistidas por computadora basadas en el uso de CDFs: ACDFs

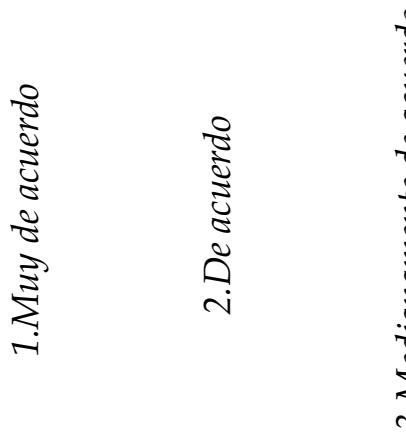

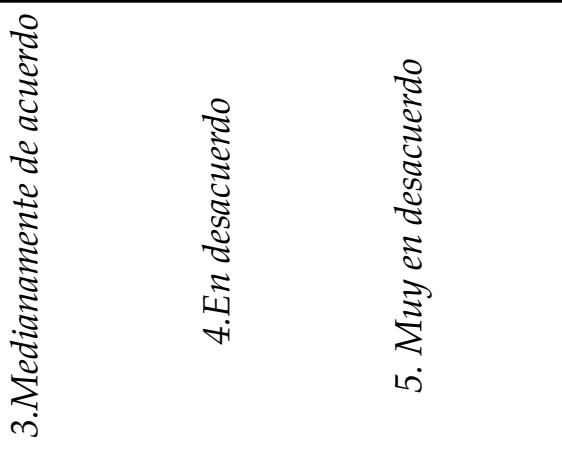

1. El trabajo en clase y extraclase complementado con el uso de CDFs le pareció útil.

2. Las ACDFs fueron difíciles.

3. Las ACDFs fueron divertidas.

4. Las ACDFs tuvieron instrucciones claras.

\begin{tabular}{|c|c|c|c|c|}
\hline $77.8 \%$ & $18.5 \%$ & $0.0 \%$ & $0.0 \%$ & $3.7 \%$ \\
\hline $11.1 \%$ & $7.4 \%$ & $51.9 \%$ & $18.5 \%$ & $11.1 \%$ \\
\hline $14.8 \%$ & $25.9 \%$ & $55.6 \%$ & $3.7 \%$ & $0.0 \%$ \\
\hline $37.0 \%$ & $48.2 \%$ & $11.1 \%$ & $3.7 \%$ & $0.0 \%$ \\
\hline $48.2 \%$ & $37.0 \%$ & $11.1 \%$ & $3.7 \%$ & $0.0 \%$ \\
\hline $44.5 \%$ & $33.3 \%$ & $22.2 \%$ & $0.0 \%$ & $0.0 \%$ \\
\hline $25.9 \%$ & $33.4 \%$ & $33.3 \%$ & $7.4 \%$ & $0.0 \%$ \\
\hline $51.9 \%$ & $33.3 \%$ & $11.1 \%$ & $0.0 \%$ & $3.7 \%$ \\
\hline $48.2 \%$ & $37.0 \%$ & $11.1 \%$ & $0.0 \%$ & $3.7 \%$ \\
\hline $59.3 \%$ & $33.3 \%$ & $3.7 \%$ & $3.7 \%$ & $0.0 \%$ \\
\hline $70.4 \%$ & $25.9 \%$ & $0.0 \%$ & $3.7 \%$ & $0.0 \%$ \\
\hline $3.7 \%$ & $0.0 \%$ & $22.2 \%$ & $25.9 \%$ & $48.2 \%$ \\
\hline
\end{tabular}

"Muy desfavorable". En el programa SPSS se generó una agrupación visual en correspondencia con esta escala valorativa. Los porcentajes encontrados se muestran en la Figura 1, donde claramente se evidencia una postura mayoritaria de un $96.3 \%$ a favor de estimar positivamente las actividades de enseñanza y aprendizaje implementadas durante el primer ciclo lectivo del año 2020, basadas en el empleo pedagógico de documentos con un formato computable.

La Figura 2 comparte una nube de palabras obtenida mediante el uso del lenguaje Wolfram, a partir de las respuestas brindadas por los alumnos con respecto a la pregunta abierta del cuestionario, donde señalaron tres fortalezas de las ACDFs. La nube es consistente con las categorías de respuesta encontradas bajo el criterio de al menos una coincidencia de opinión en un $70 \%$. Se aprecia cómo los estudiantes aprueban las actividades asistidas por computadora al permitir: resolver problemas y ejercicios, practicar y revisar resultados, y brindar una alternativa distinta para desarrollar los procesos de enseñanza y aprendizaje que contribuye con un entendimiento más benigno de los contenidos. 


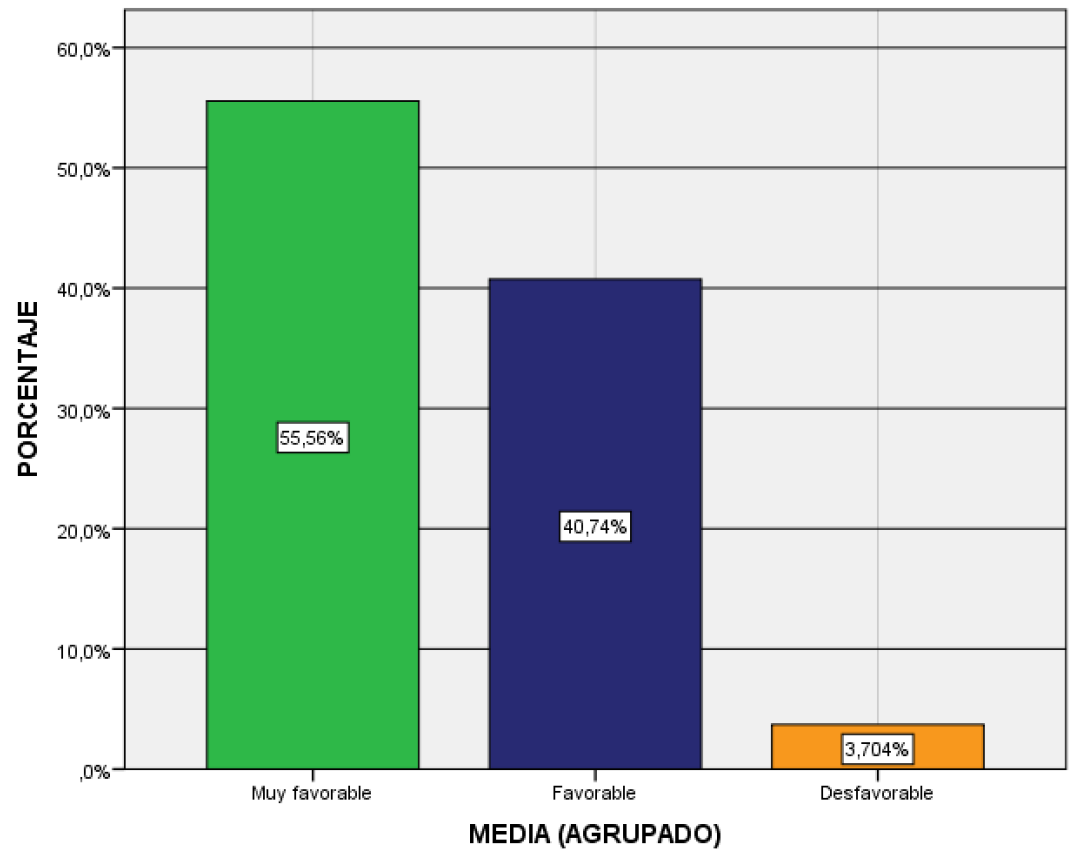

Figura 1: Porcentajes de la escala valorativa en la dimensión actividades asistidas por computadora basadas en el uso de CDFs. Fuente: Elaboración propia.

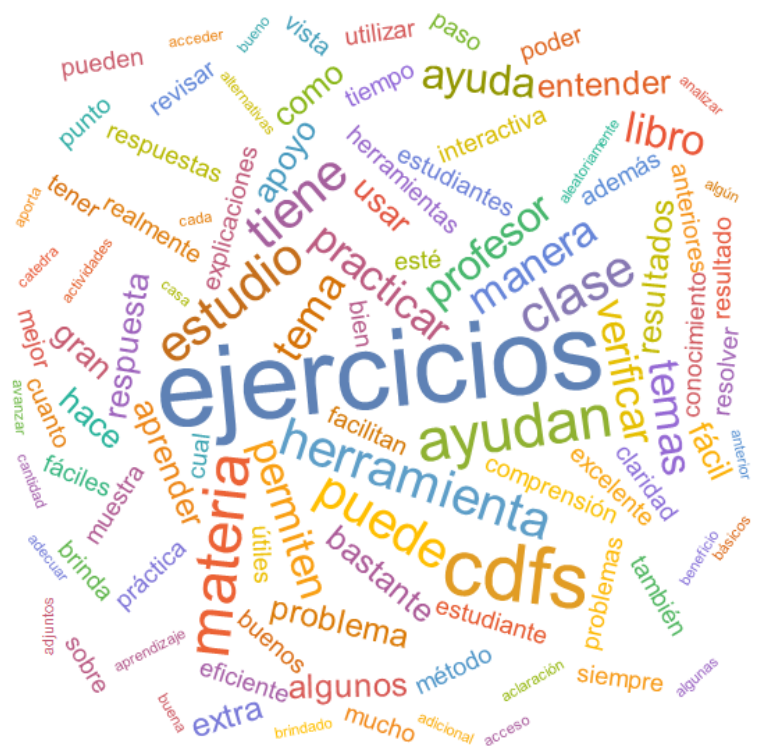

Figura 2: Nube de palabras sobre las fortalezas de las actividades asistidas por computadora basadas en el uso de CDFs. Fuente: Elaboración propia.

\subsection{Percepción estudiantil sobre la calidad de los CDFs para la enseñanza y el aprendizaje de la materia MAT030 Matemática para Informática}

Las 130 aplicaciones CDFs elaboradas por los autores de este artículo, se sometieron a escrutinio por parte de los 27 estudiantes que completaron el cuestionario administrado a finales del primer ciclo lectivo del año 2020, constituyendo lo anterior, la segunda dimensión del instrumento. Estos alumnos utilizaron los CDFs en apoyo a las lecciones de clase presenciales y también, a sus horas de estudio independiente. La Tabla 11 comparte los valores porcentuales calculados a partir de las respuestas consignadas en la escala Likert empleada. 
Tabla 11: Porcentajes escala Likert: Calidad de los CDFs para la enseñanza y el aprendizaje de la materia MAT030 Matemática para Informática. Fuente: Elaboración propia

Puntos fuertes y débiles

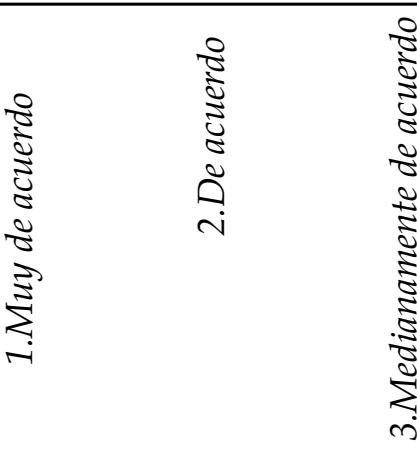

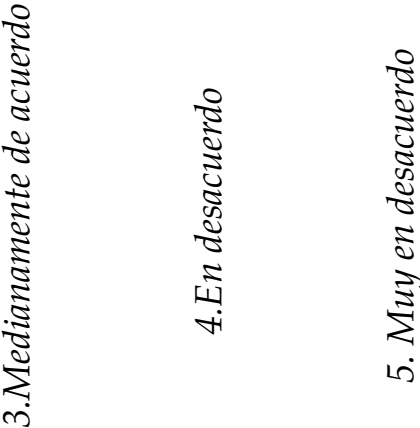

1. Los CDFs le parecieron útiles.

2. Los CDFs son de uso fácil.

3. Los CDFs son una herramienta incompleta para el curso.

4. Los CDFs motivan el estudio hacia la materia.

5. Los CDFs proveen recursos de análisis y exploración.

6. Los CDFs son aburridos.

7. Los CDFs facilitan la visualización de conceptos.

8. Los CDFs permiten analizar problemas.

9. Los CDFs permiten resolver problemas de manera adecuada.

10. Los CDFs facilitan la profundización de los contenidos.

11. Los CDFs son una herramienta propicia para el curso MAT030.

12. Los CDFs mejoran la comprensión de algoritmos.

13. Los CDFs permiten abordar el estudio de problemas con datos reales.

14. Los CDFs mejoran la comprensión conceptual.

15. Los CDFs facilitan el aprendizaje (la obtención del conocimiento).

16. Los CDFs mejoran la enseñanza (formas de explicación del docente).

17. Le agradaron los CDFs.

18 . Se tiene en general una percepción positiva de los CDFs para apoyar desde un punto de vista educativo el curso MAT030.

\begin{tabular}{|c|c|c|c|c|}
\hline $70.4 \%$ & $22.2 \%$ & $3.7 \%$ & $3.7 \%$ & $0.0 \%$ \\
\hline $40.8 \%$ & $40.7 \%$ & $18.5 \%$ & $0.0 \%$ & $0.0 \%$ \\
\hline $3.7 \%$ & $7.4 \%$ & $11.1 \%$ & $40.7 \%$ & $37.1 \%$ \\
\hline $33.4 \%$ & $33.3 \%$ & $29.6 \%$ & $3.7 \%$ & $0.0 \%$ \\
\hline $33.4 \%$ & $40.7 \%$ & $22.2 \%$ & $3.7 \%$ & $0.0 \%$ \\
\hline $0.0 \%$ & $0.0 \%$ & $25.9 \%$ & $44.4 \%$ & $29.7 \%$ \\
\hline $44.5 \%$ & $22.2 \%$ & $22.2 \%$ & $11.1 \%$ & $0.0 \%$ \\
\hline $51.9 \%$ & $33.3 \%$ & $14.8 \%$ & $0.0 \%$ & $0.0 \%$ \\
\hline $51.9 \%$ & $37.0 \%$ & $7.4 \%$ & $0.0 \%$ & $3.7 \%$ \\
\hline $37.1 \%$ & $33.3 \%$ & $14.8 \%$ & $11.1 \%$ & $3.7 \%$ \\
\hline $77.8 \%$ & $22.2 \%$ & $0.0 \%$ & $0.0 \%$ & $0.0 \%$ \\
\hline $51.9 \%$ & $22.2 \%$ & $22.2 \%$ & $3.7 \%$ & $0.0 \%$ \\
\hline $37.0 \%$ & $55.6 \%$ & $0.0 \%$ & $7.4 \%$ & $0.0 \%$ \\
\hline $44.5 \%$ & $22.2 \%$ & $33.3 \%$ & $0.0 \%$ & $0.0 \%$ \\
\hline $44.5 \%$ & $40.7 \%$ & $14.8 \%$ & $0.0 \%$ & $0.0 \%$ \\
\hline $51.9 \%$ & $25.9 \%$ & $18.5 \%$ & $3.7 \%$ & $0.0 \%$ \\
\hline $59.3 \%$ & $22.2 \%$ & $18.5 \%$ & $0.0 \%$ & $0.0 \%$ \\
\hline $77.8 \%$ & $18.5 \%$ & $3.7 \%$ & $0.0 \%$ & $0.0 \%$ \\
\hline
\end{tabular}


Al igual que en la dimensión analizada en la sección que precede, se escogieron como percepciones positivas, aquellas donde la suma porcentual en las valoraciones " $1=$ Muy de acuerdo" y " $2=$ De acuerdo", dio como resultado un número mayor o igual a $70 \%$. En consecuencia, predominan como juicios positivos sobre la calidad de los CDFs, los siguientes: los CDFs son útiles (92.6\%), son de uso fácil $(81.5 \%)$, son una herramienta completa para el curso $(77.8 \%)$, proveen recursos de análisis y exploración $(74.1 \%)$, no son aburridos $(74.1 \%)$, permiten analizar problemas $(85.2 \%)$, los CDFs permiten resolver problemas de manera adecuada (88.9\%), facilitan la profundización de los contenidos $(70.4 \%)$, son una herramienta propicia para el curso MAT030 (100\%), los CDFs mejoran la comprensión de algoritmos $(74.1 \%)$, permiten abordar el estudio de problemas con datos reales $(92.6 \%)$, facilitan el aprendizaje $(85.2 \%)$, mejoran la enseñanza $(77.8 \%)$, agradaron a su población objetivo $(81.5 \%)$, se tiene en general una percepción positiva de los CDFs para apoyar desde un punto de vista educativo el curso MAT030 (96.3\%). Además, se rescatan como percepciones negativas: los CDFs no motivan el estudio hacia la materia (33.3\%), los CDFs no facilitan la visualización de conceptos $(33.3 \%)$, los CDFs no mejoran la comprensión conceptual (33.3\%).

En estos resultados podría parecer paradójico que los participantes señalen una mejoría en la profundización de los contenidos, la comprensión algorítmica, la resolución de problemas, en el aprendizaje y en la enseñanza, pero, por otro lado, consideren que las aplicaciones CDFs son insuficientes con respecto a favorecer mayores comprensiones conceptuales de los temas de la materia MAT030. También, podría lo anterior, arrojar dudas de consistencia con respecto a los resultados inferidos en las pruebas estadísticas Mann-Whitney ya compartidas. En este punto, no se debe olvidar que el cuestionario administrado a los GE tuvo como principal objetivo recopilar únicamente percepciones de opinión, que pueden estar alejadas de una realidad medible de forma precisa. Además, los investigadores reconocen en la población estudiantil una visión de la educación matemática netamente utilitaria, donde lo que prevalece es el aprendizaje de procedimientos, esquemas de pensamiento tipo molde y formas de resolución a través de casos reproducibles en situaciones similares. Desafortunadamente, la enseñanza de una matemática integral donde la teoría constituya el insumo esencial para abordar sus aplicaciones y la ejecución de ejercicios prácticos muchas veces es sustituida en las instituciones de enseñanza superior, por enfoques, implícitos en las estrategias de aprendizaje de los alumnos, que dan mayor valía a la reproducción sin sentido (Vílchez, 2014).

Por otra parte, en la Figura 3 se socializan los valores porcentuales encontrados al realizar una medición general de las respuestas en la escala Likert, vinculada con la dimensión "calidad de los CDFs para la enseñanza y el aprendizaje de la materia MAT030". La metodología utilizada fue la misma descrita en la sección anterior, creando una variable MEDIA en el software SPSS junto con una agrupación visual para cada una de las medias aritméticas " $\mathrm{n}$ " por registro, en reciprocidad a la escala: $1 \leq n<2$ : "Muy favorable", $2 \leq n<3$ : "Favorable", $3 \leq n<4$ : "Desfavorable" y $4 \leq n \leq 5$ : "Muy desfavorable". Se aprecia en la Figura 3, cómo un $96.3 \%$ de la muestra, está a favor de considerar de manera positiva la calidad de los CDFs diseñados para el curso MAT030.

El cuestionario aplicado a los 27 estudiantes de los grupos experimentales incluyó dos preguntas abiertas con la intención de determinar fortalezas y debilidades reveladas en los documentos con un formato computable elaborados para el curso MAT030. En la Figura 4 se observa una nube de palabras relacionada con las fortalezas detectadas por la muestra y en la Figura 5 una nube de palabras asociada con las debilidades manifestadas. Ambas nubes de palabras se gestaron en el software Wolfram Mathematica pasando de manera directa las respuestas de opinión de los alumnos participantes. Entre las fortalezas recurrentes con un $70 \%$ de aparición, se destacan: eficientes herramientas para el estudio individual, permiten verificar resultados paso a paso y respuestas directas sin depender del docente, siempre están disponibles (localmente o en la web), son claros y sencillos de usar, permiten practicar los temas del curso con ejercicios pseudoaleatorios y son un excelente complemento educativo abriendo nuevas formas de abordaje de todos los contenidos de la materia MAT030. En lo referente a las debilidades identificadas con un $70 \%$ de coincidencia, los alumnos señalaron: se requiere el uso y conocimiento de símbolos muy específicos en algunos CDFs para procesar la información deseada e interpretar algunos datos de salida, los CDFs en la web tienden a tardar mucho en su respuesta o eventualmente a congelarse, los CDFs podrían fomentar el plagio y generar cierto grado de dependencia 
si se abusa de su utilización al evitar el uso de procedimientos manuales.

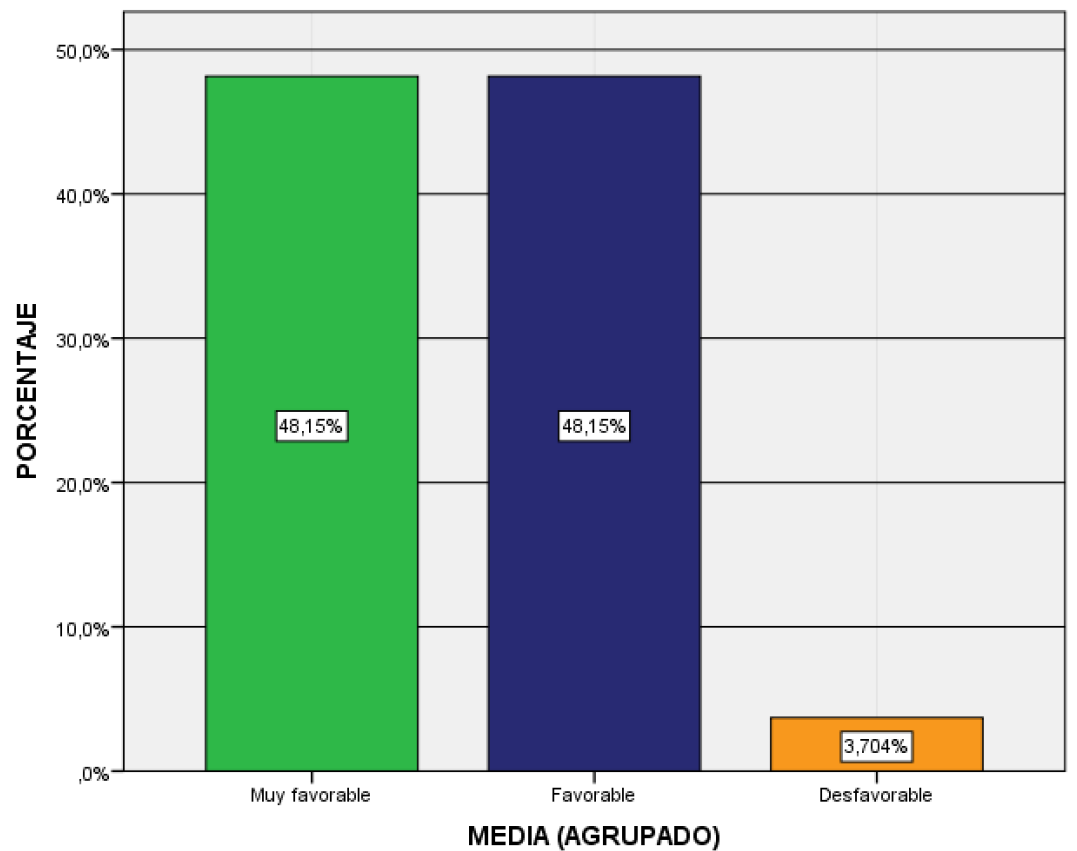

Figura 3: Porcentajes de la escala valorativa en la dimensión calidad de los CDFs para la enseñanza y el aprendizaje de la materia MAT030. Fuente: Elaboración propia.

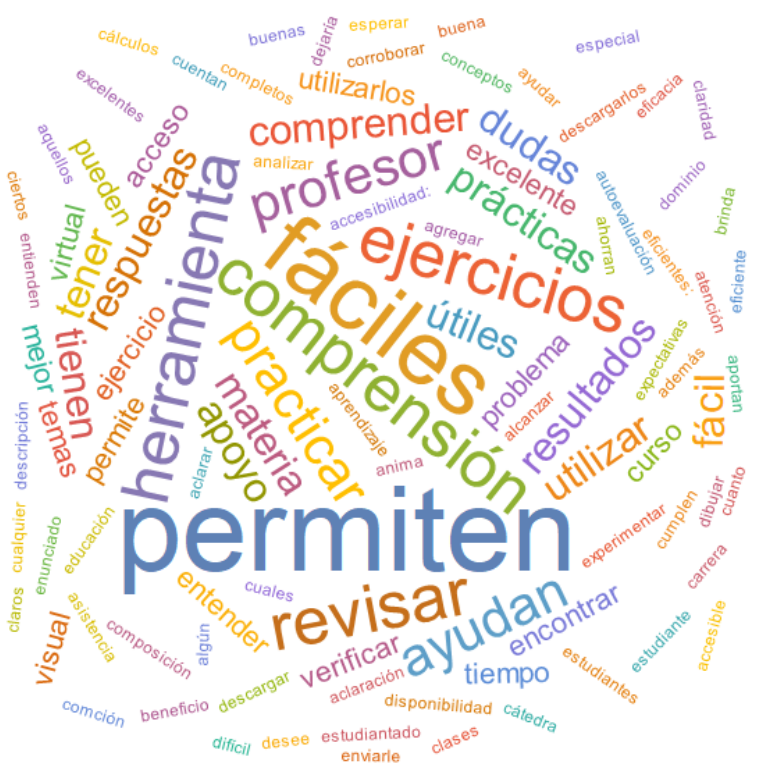

Figura 4: Nube de palabras sobre las fortalezas de los CDFs para la enseñanza y el aprendizaje de la materia MAT030. Fuente: Elaboración propia.

\subsection{Comparación de rendimiento académico entre los grupos experimentales y el grupo de control}

Los grupos experimentales y los grupos de control se compararon en términos del rendimiento académico mostrado al finalizar el primer ciclo lectivo 2020. Todos ellos, realizaron las mismas pruebas parciales ponderando un $90 \%$ de la nota final y el $10 \%$ restante se asignó a tres quices planificados por los docentes responsables de los grupos, pero bajo las mismas directrices de la cátedra MAT030, garantizándose así en las pruebas cortas, cierto grado de homogeneidad. 


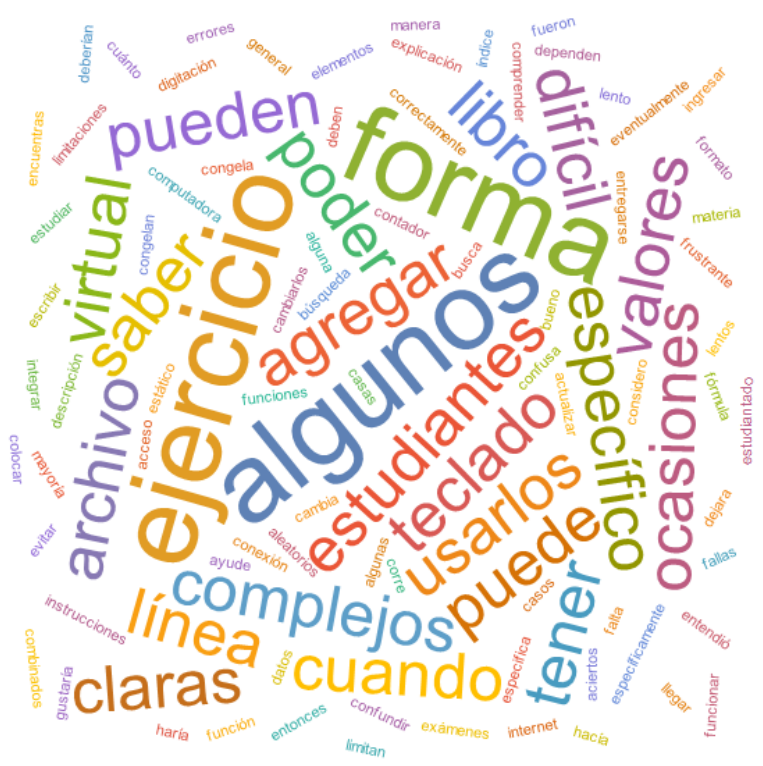

Figura 5: Nube de palabras sobre las debilidades de los CDFs para la enseñanza y el aprendizaje de la materia MAT030. Fuente: Elaboración propia.

Los registros de las notas finales de los estudiantes en los GE y los GC sirvieron de base para implementar en el software SPSS una prueba de hipótesis paramétrica $t$ de Student en muestras independientes comparando las medias (Hurtado y Hurtado, 2015). Si la diferencia entre las medias en los GE y los GC es reducida, se acepta la hipótesis nula Ho: "no hay diferencia en el rendimiento académico entre los GE y los GC", frente a la hipótesis de investigación Ha: "es más favorable el rendimiento académico en los GE". La información procesada por el programa estadístico SPSS aparece en la Tabla 12.

Tabla 12: Prueba t de Student: Comparación de rendimiento académico. Fuente: Elaboración propia

\begin{tabular}{llllllll}
\hline & $t$ & $g l$ & $\begin{array}{l}\text { Sig. (bi- } \\
\text { lateral) }\end{array}$ & $\begin{array}{l}\text { Diferencia } \\
\text { de medias }\end{array}$ & $\begin{array}{l}\text { Error tip. } \\
\text { de la dife- } \\
\text { rencia }\end{array}$ & $\begin{array}{l}95 \% \text { Intervalo de } \\
\text { confianza para la di- } \\
\text { ferencia }\end{array}$ \\
\hline $\begin{array}{l}\text { Se han asumido } \\
\text { varianzas iguales } \\
\text { en las medias }\end{array}$ & -2.233 & 86 & 0.028 & -0.83545 & 0.37415 & -1.5792 & -0.09167 \\
$\begin{array}{l}\text { No se han asu- } \\
\text { mido varianzas } \\
\text { iguales en las } \\
\text { medias }\end{array}$ & & & & & & & \\
\hline
\end{tabular}

La columna Sig. (bilateral) en la Tabla 12, evidencia que el valor de significancia " $\rho$ " es menor que 0.05, el mínimo aceptable bajo el supuesto de un $5 \%$ de error. Por lo tanto, se infiere que la diferencia entre las medias de rendimiento académico de los GE y los GC, respectivamente, no es aleatoria, aceptándose la hipótesis alternativa Ha. Es decir, en este estudio de caso se podría interpretar que existe un mejor rendimiento en las notas finales de los estudiantes pertenecientes a los grupos experimentales, que sí utilizaron los documentos con un formato computable en los procesos de enseñanza y aprendizaje del curso MAT030 Matemática para Informática. Cabe aclarar, que dicha tendencia no se puede atribuir exclusivamente al empleo de los CDFs como un recurso didáctico. 


\section{Conclusiones}

La presente investigación ha comprobado mediante la realización de cinco pruebas de hipótesis no paramétricas Mann-Whitney en los grupos experimentales participantes, una mayor comprensión conceptual en comparación con la cantidad de conceptos asimilados por los grupos de control en los cinco ejes temáticos de contenido principales de la materia MAT030 Matemática para Informática, específicamente: lógica proposicional, teoría de números, teoría de conjuntos, inducción y progresiones, y teoría de funciones.

La experiencia de implementación de uso de los CDFs como apoyo complementario a los procesos de enseñanza y aprendizaje del curso MAT030, resultó ser exitosa tanto en las actividades didácticas (ACDFs) llevadas a cabo en clase, así como también, en la calidad de las 130 aplicaciones diseñadas con un formato computable.

Las ACDFs fueron útiles, con instrucciones claras, agradables, formativas en términos de aprendizaje, apropiadas en su organización temporal y llenaron las expectativas de la población objetivo. En ellas, se encuentra una posibilidad de mejora por parte de los investigadores, al requerir equilibrar sus niveles de dificultad en atención al hecho de que la materia MAT030 responde a estudiantes de primer ingreso de la carrera Ingeniería en Sistemas de Información de la Universidad Nacional de Costa Rica. Estas actividades ofrecen alternativas de mediación complementarias al enfoque que típicamente se ha venido empleando en este contexto educativo y de allí la importancia de su riqueza metodológica y de su continuidad en futuras versiones de otros ciclos lectivos.

Los documentos con un formato computable creados para el curso MAT030 se caracterizaron por: ser de uso fácil, una herramienta completa, proveer recursos de análisis y exploración, no ser aburridos, facilitar mecanismos para analizar y resolver problemas, profundizar los contenidos de la materia y mejorar la comprensión procedimental, el aprendizaje y la enseñanza. Como elementos de depuración para los CDFs se destaca la necesidad de incluir instrucciones dentro de estas aplicaciones que puntualicen más apropiadamente el simbolismo requerido para su uso y la interpretación de los datos de salida que proporcionan. Los CDFs representan una oportunidad didáctica innovadora en una cátedra donde ha prevalecido un esquema de enseñanza basado en recursos no computacionales. Su valía en este sentido, demanda cambios oportunos en las prácticas pedagógicas y desafíos didácticos que pueden conducir en el mejor de los casos, a aprendizajes más significativos y duraderos.

El empleo de CDFs como complemento a los procesos educativos en la materia MAT030 Matemática para Informática, no está exento de riesgos en un escenario donde el sistema de evaluación se mantenga con un formato tradicional, dado que las poblaciones de alumnos podrían cometer el error de utilizar mal estos recursos digitales: sea para propiciar fraude en las pruebas escritas, o bien, para omitir la realización de cálculos manuales en sus horas de estudio individual. Como recomendación futura, valdría la pena en la Escuela de Matemática de la UNA, dar cabida a la apertura de grupos del curso MAT030 con un sistema de evaluación diferenciado y en sincronía con el empleo de documentos con un formato computable.

En la presente investigación se comprobó, además, un aprovechamiento académico más favorable en los grupos experimentales al ser comparados con los grupos de control. Si bien es cierto, esto no es una garantía generalizable, de alguna manera corrobora la importancia de buscar y fortalecer, dentro de la educación matemática y particularmente, dentro de la cátedra del curso MAT030, distintas alternativas didácticas que promuevan atender necesidades de aprendizaje con matices diversificados.

Este trabajo siendo una extensión de lo propuesto por Vílchez y Ávila (2021), corrobora de manera similar, pero en este caso en la población estudiantil, los resultados preliminares que desde ese momento se arrojaban a la luz de la opinión de los docentes que formaban parte de la cátedra MAT030, respecto a la calidad positiva del libro "Matemática para ingeniería mediante el uso de CDFs" (Vílchez y Ávila, 2020), los documentos con un formato computable elaborados para este curso y la necesidad de un cambio en las formas de enseñanza tradicionales. 
No todos los alumnos aprenden de la misma manera y ser consciente de ello, es la premisa que en el mejor de los casos orientará una enseñanza más equitativa. La tecnología educativa, a este respecto, ha servido en el contexto de esta pesquisa, como un puente de transformación, disrupción e innovación, intrínseco en un marco de planeamiento pedagógico crítico, constructivo y flexible.

\section{Reconocimientos}

Este trabajo fue elaborado en el marco del proyecto en docencia inscrito en la Universidad Nacional de Costa Rica, que llevó por título: "CDFs como recurso didáctico para apoyar la materia MAT030 Matemática para Informática", código SIA: 0126-18.

\section{Bibliografía}

[1] Artigue, M. (2013). La educación matemática como un campo de investigación y como un campo de práctica: resultados, desafíos. Revista Cuadernos de Investigación y Formación en Educación Matemática, 8(11), 43-59. Recuperado de https://revistas.ucr.ac.cr/index.php/cifem/issue/view/1518.

[2] Ávila, J. y Vílchez, E. (Noviembre, 2019). Lógica y teoría de conjuntos mediante diagramas de Venn-Euler por sectores. En D. Sánchez (Presidencia), VI Jornadas Iberoamericanas de Innovación Educativa en el Ámbito de las TIC y las TAC. Congreso con sede en España, Universidad de Las Palmas de Gran Canaria. Recuperado de https://accedacris.ulpgc.es/handle/10553/58081.

[3] Cantoral, R. (2016). Teoría socioepistemológica de la matemática educativa. México: Gedisa.

[4] Castillo, S. (2008). Propuesta pedagógica basada en el constructivismo para el uso óptimo de las TIC en la enseñanza y el aprendizaje de la matemática. Revista Relime, 11(2), 171194. Recuperado de http://www.scielo.org.mx/pdf/relime/v11n2/v11n2a2.pdf.

[5] Berlanga, V. y Rubio, M. (2012). Clasificación de pruebas no paramétricas. Cómo aplicarlas en SPSS. Revista REIRE, 5(2), 101-113. doi:http:/ /dx.doi.org/10.1344/reire2012.5.2528.

[6] García, A. (2014). Estrategia metodológica para la elaboración y utilización de objetos de aprendizaje interactivos y experimentales en el proceso de enseñanzaaprendizaje de la Matemática Discreta en la UCI (Tesis de maestría, Universidad de la Habana, Facultad de Matemática y Computación). Cuba. Recuperada de https:/ /www.researchgate.net/publication/286231798.

[7] Gerver, R. (2013). Crear hoy la escuela del mañana: la educación y el futuro de nuestros hijos. México: Ediciones SM.

[8] Hernández, R., Fernández, C. y Baptista, P. (2006). Metodología de la investigación. México: McGraw-Hill Interamericana.

[9] Hurtado, A. L. y Hurtado, C. L. (2015). La toma de decisiones en investigación educativa con SPSS. Recuperado de http:/ /www.qartuppi.com/2015/SPSS.pdf.

[10] Penrose, R. (1991). La nueva mente del emperador. Barcelona: Grijalbo Mondadori.

[11] Resnick, L. y Ford, W. (1990). La enseñanza de las matemáticas y sus fundamentos psicológicos. España: Paidós.

[12] Robinson, K. (2015). Escuelas creativas: la revolución que está transformando la educación. USA: Vintage. 
[13] Vílchez, E. (2014). Estrategias de enseñanza para el curso EIF-203 Estructuras discretas para informática a través del uso de las redes sociales Facebook y Twitter. Revista Electrónica Educare, 18(2), 39-70. doi: http://dx.doi.org/10.15359/ree.18-2.3.

[14] Vílchez, E. (2019). Estudio de caso: Estrategia de enseñanza y aprendizaje asistida por computadora para un curso de matemática discreta a través del uso del paquete VilCretas en el software Wolfram Mathematica. Revista Electrónica Educare, 23(2), 1-25. doi: https://doi.org/10.15359/ree.23-2.13

[15] Vílchez, E. y Ávila, J. (2019). Desarrollo de documentos con un formato computable utilizando el software Wolfram Mathematica. Revista digital Matemática, Educación e Internet, 20(1), 1-29. Recuperado de https://tecdigital.tec. ac.cr/revistamatematica/ Secciones/Didactica_y_Software/RevistaDigital_FAvila_V20_n1_2019/index.html.

[16] Vílchez, E. y Ávila, J. (2020). Matemática para ingeniería mediante el uso de CDFs. Colombia: Editorial Alpha.

[17] Vílchez, E. y Ávila, J. (2021). Enseñanza y aprendizaje de la matemática para informática empleando documentos con un formato computable (CDFs): una percepción docente en la Universidad Nacional de Costa Rica. Revista digital Matemática, Educación e Internet, 21(2), 1-17. 